\title{
Clinical Management
}

\section{Il direttore di "Struttura Complessa di nefrologia e/o dialisi": tra l'incudine ed il martello. Il rapporto con i soggetti-incudine "collaboratori".}

G. Carnevali*, G. Quintaliani**

${ }^{*}$ Esperto in legislazione ed organizzazione sanitaria

${ }^{* *}$ Coordinatore Governo Clinico della SIN

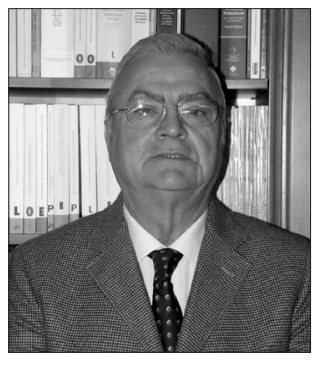

Gianfranco Carnevali

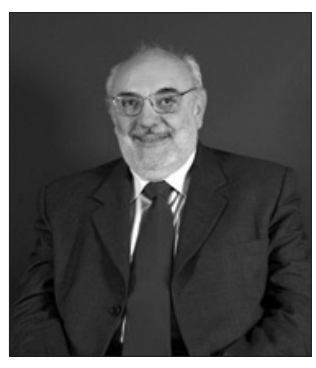

Giuseppe Quintaliani

\section{Premessa}

Ricordato che nell'articolo Il direttore di "Struttura Complessa di nefrologia e/o dialisi”: tra l'incudine ed il martello. Il rapporto con i soggetti-martello, pubblicato nel precedente numero di questa rivista, Carnevali aveva:

- sottolineate le difficoltà in cui, spesso, si trova ad operare ciascun direttore nello svolgimento delle competenze e responsabilità connesse all'incarico,

- individuato due categorie di soggetti-incudine cioè gli operatori assegnati alla struttura (i collaboratori del direttore) e quanti ad essa si rivolgono per ottenere soddisfazione ai propri bisogni di salute (l'utenza),

ci accingiamo ad esplecitare gli elementi che il direttore deve opportunamente tenere presenti nella gestione del rapporto con i collaboratori rilevando che l'affermazione concernente le riferite difficoltà trova piena validità soprattutto in tale contesto e rinviando a specifico successivo intervento l'approfondimento del rapporto direttore/utenza.

Ai fini del presente contesto preme sottolineare che ogni direttore deve sia dirigere ed organizzare la struttura nel rispetto dei contenuti dell'atto aziendale (ad esempio, articolazione in strutture semplici, afferenza dipartimen- tale, modalità di funzionamento) che dotarla di spazi, attrezzature e personale quali-quantitativamente idonei a raggiungere gli obiettivi formalizzati nel budget definito in contradditorio con il direttore generale dell'azienda, ferma restando la doverosità di contemperare i diritti/ le aspettative dei collaboratori con il modello organizzativo/funzionale prefigurato per soddisfare le aspettative e, a volte, i diritti dell'utenza.

All'enunciato approfondimento procediamo con riferimento:

- alla gestione,

- allo sviluppo,

- alla responsabilizzazione

degli operatori assegnati alla struttura complessa, cioè dei collaboratori del singolo direttore.

\section{La gestione dei collaboratori ${ }^{(1),(2)}$}

\section{Aspetti generali ${ }^{(1.1)}$}

Per poter svolgere adeguatamente la gestione dei collaboratori -da considerare come doverosa competenza di ogni direttore finalizzata all'utilizzazione di ciascuno di essi in modo funzionale rispetto al raggiungimento degli obiettivi della struttura, cioè al soddisfacimento dei bisogni sanitari della popolazione di riferimento- ogni direttore deve essere consapevole:

- del ruolo centrale che il personale assume per l'erogazione di prestazioni sia della migliore qualità possibile che appropriate, cioè di prestazioni efficaci avendo presente che, nelle aziende/imprese che producono ed erogano servizi, la qualità di queste è direttamente proporzionale alla qualità degli operatori chiamati a farsene carico ed il fattore "personale" assume preponderante importanza rispetto agli altri due fattori (capitale ed or- 
ganizzazione) da utilizzare per perseguirne gli scopi e, conseguentemente, forte valenza strategica;

di dover:

- evitare l'insorgere di conflittualità all'interno della struttura, sapendo che esse incidono negativamente sul suo funzionamento;

- garantire la funzionalità della struttura conciliandone, ove possibile, le esigenze con le aspettative personali, familiari, sociali dei collaboratori;

- tenere conto delle direttive fornite dalla struttura preposta all'amministrazione del personale -di norma in line alla direzione strategica aziendale- cui spetta elaborare ed enunciare i principi da osservare per una corretta ed uniforme applicazione delle disposizioni legislative, contrattuali, a volte, regolamentari degli istituti rientranti nella gestione del personale;

- acquisire le capacità manageriali (ad esempio, leadership, terzietà nei sistemi di valutazione/premialità, disponibilità a supportare chi manifesta difficoltà, abilità negoziatorie, motivazionali, di guida) complementari all'auspicato possesso di quelle cosiddette innate (ad esempio, carisma, autorevolezza, sensibilità, empatia);

- mettere a disposizione del personale un trasparente e tempestivo sistema informativo interno alla struttura;

- verificare le attitudini personali del singolo collaboratore onde utilizzarlo in coerenza con le stesse;

- definire modelli organizzativi idonei in grado di favorire il raggiungimento degli obiettivi sia di ciascun collaboratore che della struttura complessivamente considerata.

\section{Modelli applicativi dei più importanti istituti rientranti nella gestione $\mathrm{e}^{(1.2)}$}

La gestione dei collaboratori, quale funzione di maggiore rilevanza e delicatezza del management, impone al direttore di organizzare l'attività della struttura tenendo conto:

- delle disposizioni legislative e contrattuali, compresi i contratti integrativi aziendali, che disciplinano gli istituti ad essa riconducibili;

- dei diritti e, ove possibile, delle aspettative degli operatori;

- delle esigenze della popolazione di riferimento;

- della necessità di non farsi condizionare né da sollecitazioni di terzi né da esigenze proprie o dei collaboratori.

Allo scopo egli, tra l'altro, deve:

- definire gli orari di servizio, cioè di funzionamento, di tutte le attività della struttura, onde assicurare che gli orari di lavoro del personale siano coerenti con esse e, quindi, con i predefiniti orari di servizio;

- assicurare a ciascun collaboratore un luogo sicuro/ confortevole, adeguate tecnologie, orari di lavoro compatibili, ove possibile, con le proprie esigenze familiari/personali;

- applicare alcuni istituti legislativi e contrattuali (ad esempio: ferie/festività soppresse; riposo settimanale; festività; reperibilità/pronta disponibilità; recupero maggiori orari prestati; lavoro straordinario; assenze per maternità, malattia, infortunio; permessi sindacali, per svolgere cariche pubbliche, per partecipazione a congressi/convegni/corsi, per donazione del sangue, per nascita di figli, per matrimonio) nel rispetto delle direttive formalizzate dalla S.C. "Amministrazione del Personale", cui spetta peraltro applicare numerosi altri istituti (ad esempio, mobilità interna, comandi e distacchi, congedi per partecipare a corsi di formazione, aspettative, benefici per i portatori di handicap o in particolari condizioni fisiche).

Degli istituti "ferie/festività soppresse", "permessi per partecipazione dei dirigenti medici a congressi, convegni, corsi", "riserva oraria settimanale destinata ad attività non assistenziali", "lavoro straordinario", "servizi di guardia e pronta disponibilità", "partecipazione ai procedimenti e provvedimenti disciplinari", "partecipazione alle procedure per l'affidamento degli incarichi ai collaboratori medici", di seguito forniamo elementi utili per una funzionale applicazione onde evidenziarne le relative peculiarità.

\subsection{Ferie/festività soppresse $e^{(1.2)}$}

Il direttore, tenuto conto degli orari di servizio predeterminati per lo svolgimento delle attività della struttura, deve indicare le presenze minime di cui essa deve disporre, per ciascun profilo professionale, nei vari periodi dell'anno (ordinariamente, in occasione di festività natalizie/pasquali, diminuzione/aumento dei potenziali fruitori nei periodi di ferie/vacanze) onde consentire ai collaboratori di accordarsi sui rispettivi periodi di assenza, fermo restando, nel caso di mancata intesa, l'obbligo di provvedervi direttamente per i dirigenti e da parte del caposala per il personale addetto all'assistenza. L'incombenza gli impone di sapere che: a. le ferie:

a.1. spettano per:

- 32 giorni lavorativi annui (30 nel primo triennio di servizio), ovvero per 28 e 26 quando l'orario di lavoro sia articolato su 5 giorni, di cui 2 sostitutivi "ex art. 1. comma 1, lettera a), della legge n. 937/1997- di festività soppresse, 
- ulteriori 15 giorni ai sottoposti a radiazioni ionizzanti, esse vanno fruite obbligatoriamente durante l'anno di riferimento, in relazione allo scopo della concessione;

a.2. sono ridotte proporzionalmente ai dodicesimi di servizio prestati calcolandosi come mese intero la frazione superiore a 15 giorni;

a.3. sono irrinunciabili e non riducibili per assenze per malattia ed infortunio;

a.4. vanno concesse per un periodo minimo continuativo di 15 giorni durante il periodo estivo;

a.5. non sono più monetizzabili, nemmeno nel caso in cui -come prevedono le disposizioni contrattualiall'atto della cessazione dal servizio risulti che il mancato godimento consegua da esigenze di servizio ovvero da cause non attribuibili all'interessato, in relazione al disposto dell'art. 4 , comma 8 , del decreto legge 6 luglio 2012, n. 95, convertito con modificazioni in legge 7 agosto 2012, n. 135 sulla base del quale:

- le ferie, i riposi ed i permessi spettanti al personale, anche di qualifica dirigenziale, delle PP.AA. vanno fruite obbligatoriamente e non danno luogo in ogni caso alla corresponsione di trattamenti economici sostitutivi,

- la citata disposizione si applica anche in caso di cessazione del rapporto di lavoro per mobilità, dimissioni, risoluzione, pensionamento e raggiungimento del limite di età, con conseguente disapplicazione delle eventuali disposizioni normative e contrattuali più favorevoli,

- a violazione del disposto in questione, oltre a comportare il recupero delle somme indebitamente erogate, è fonte di responsabilità disciplinare ed amministrativa per il dirigente responsabile;

b. Le festività soppresse -ex art. 1, comma 1, lettera b), della citata legge n. 937/1997- comportano la concessione anche di 4 giornate aggiuntive di riposo le quali vanno monetizzate con l'erogazione di $€$ 4,39 ciascuna, se non sono fruite entro il mese di gennaio dell'anno successivo a quello di riferimento. La concessione va autorizzata solo dopo l'esaurimento delle ferie riferite all'anno precedente.

\subsection{Permessi per partecipazione dei dirigenti medici a congressi, convegni e corsi vari ${ }^{(1.2)}$}

La materia è disciplinata dall'art. 23 del cccl sottoscritto il 5 dicembre 1996 il quale prevede che detti dirigenti possano ottenere al massimo 8 giorni all'anno di permessi retribuiti per partecipare a convegni, congressi o corsi di aggiorna- mento, perfezionamento o specializzazione professionale facoltativi connessi all'attività di servizio, senza fornire indicazioni circa le modalità procedurali da realizzare per chiederli ed autorizzarli. La lamentata carenza, salvo suo recupero in sede di contrattazione integrativa aziendale, rende opportuno rivolgersi alcune domande per farsi carico subito dopo di fornire risposte che consentano di prefigurare una procedura accettabile e cioè chiedersi:

- da un lato che sia tenuto a:

a) decidere circa la connessione o meno tra l'oggetto del convegno, congresso o corso e l'attività di servizio ovvero se le esigenze di servizio consentano o meno di autorizzare la partecipazione;

b) concedere l'autorizzazione nei casi in cui i richiedenti/aspiranti siano in numero superiore rispetto a quello da autorizzare senza creare disfunzioni alla struttura;

c) stabilire se l'autorizzazione richiesta da chi è chiamato come "relatore" ovvero come "inviter speaker" debba avere priorità rispetto a chi intenda parteciparvi come "uditore";

- da un altro lato se la mancata concessione del permesso retribuito, consenta di chiedere ferie considerandone scontata l'autorizzazione e se debba considerarsi scontata la partecipazione del direttore a tutte le iniziative che abbiano oggettiva connessione con le attività della struttura.

Prima di fornire risposta a ciascuna delle domande ipotizzate riteniamo necessario richiamare l'attenzione sul

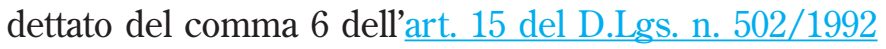
e smi, secondo il quale il direttore di struttura complessa medica deve svolgere "oltre a quelle derivanti dalle specifiche competenze professionali, funzioni di direzione $e$ organizzazione della struttura ... e l'adozione delle decisioni necessarie per il corretto espletamento del servizio e per realizzare l'appropriatezza degli interventi" e nel contempo "è responsabile dell'efficace ed efficiente gestione della risorse attribuite" alla stessa, per sottolineare che tali delicate competenze e responsabilità dovrebbero indurlo a decisioni basate esclusivamente su valutazioni oggettive onde evitare che valutazioni basate su criteri diversi gli precludano di raggiungere i citati obiettivi e, quindi, mettano in discussione la prosecuzione nello svolgimento dell'incarico; sempre che non auspichi (ovvero sia certo) che anche la sua valutazione sarà comunque positiva!

Ciò premesso ci sembra di poter affermare che:

- le decisioni sugli interrogativi esplicitati sotto le lettere "a" e "b" spettano al direttore, scontata la necessità della loro motivazione e della comunicazione a tutti gli interessati;

- l'opportunità di considerare prioritario autorizzare la partecipazione di chi sia chiamato a svolgere il ruolo 
di "relatore" o di "inviter speaker" trova giustificazione in considerazione sia del prestigio che ne deriverà alla struttura sia dell'impegno professionale aggiuntivo di cui il prescelto deve farsi carico per potersi esprimere in modo adeguato, onde evitare a sé ed alla struttura brutte figure, considerati gli ambiti certamente non circoscritti della risonanza dell'incarico svolto. Ciò premesso rileviamo la necessità che i collaboratori diano tempestiva notizia al direttore dellincarico al quale sono stati chiamati sia per ottenerne un consenso preventivo di massima sia per consentirgli di rivisitare l'organizzazione delle attività della struttura, onde evitare disfunzioni al loro svolgimento, quindi penalizzazioni all'utenza, rilevando per quanto concerne:

- la richiesta di ferie conseguente alla mancata concessione del permesso retribuito, che la concessione non può considerarsi scontata in quanto questa presuppone che il direttore valuti la compatibilità dell'assenza con le obiettive esigenze di funzionalità della struttura;

- la scontata partecipazione del direttore a tutte le iniziative oggettivamente connesse alle attività della struttu$r a$, che il comportamento non sembra giustificabile, se non altro perché potrebbe far pensare che egli, da un lato, non possieda la professionalità richiesta per svolgere l'incarico e, da un altro lato, non si curi dello sviluppo dei collaboratori che, invece, dovrebbe costituire, come successivamente vedremo, compito doveroso per un idoneo svolgimento dell'incarico.

In conclusione ed in aggiunta sembra opportuno sollecitare ogni direttore ad indire all'inizio di ciascun anno specifica riunione con tutti i propri collaboratori medici onde, tenuto conto delle iniziative di interesse della struttura, definire, se possibile concordemente, chi e per quale motivo debba rendersi disponibile a parteciparvi; durante la stessa riunione sarebbe opportuno che gli interessati riferiscano come intendano utilizzare la riserva oraria di cui al successivo punto " 2.3 " che ritengono di non svolgere settimanalmente ma di cumulare onde verificarne la compatibilità con la partecipazione alle iniziative rientranti nel presente istituto contrattuale e, quindi, con le esigenze di servizio. Peraltro riteniamo che debba considerarsi scontato che i prescelti al ritorno debbano farsi carico di riferi$\mathrm{re}$, in altra specifica riunione plenaria, i contenuti dei vari temi trattati e le possibilità del recupero delle conclusioni esplicitate al fine del miglioramento dell'attività svolta.

\subsection{Riserva oraria settimanale destinata ad attività non assistenziali}

La riserva oraria in questione -ex art. 14, comma 4 , del ccnl sottoscritto il 3 novembre 2005- va utilizzata settima- nalmente ovvero, laddove le necessità di servizio non lo consentono, cumulativamente in ragione annua per l'aggiornamento professionale, la partecipazione ad attività didattiche, la ricerca finalizzata e per l'aggiornamento facoltativo cioè, -ex art. 33, comma 6 , del ccnl sottoscritto il 5 dicembre 1996- per tutte quelle iniziative selezionate discrezionalmente e documentate debitamente dai dirigenti medici interessati che non comportino oneri per l'azienda. Essa, se non utilizzata, non può essere, comunque, remunerata. Sulla base della disciplina richiamata riteniamo che la riserva oraria:

- debba considerarsi svolta settimanalmente salvo vi ostino documentate e formalizzate necessità di servizio; - debba essere preventivamente programmata in tutti i casi in cui costituisca cumulo di riserve orarie settimanali non utilizzate onde consentire al direttore di adottare le decisioni necessarie ad evitare che l'assenza dia luogo a disfunzioni nello svolgimento dell'attività della struttura; - sia aggiuntiva ai permessi di cui al precedente punto 2.2.

\subsection{Lavoro straordinario ${ }^{(1.2)}$}

Premesso che, salvo emergenze ed imprevisti, il riconoscimento di maggiori orari prestati ai fini della monetizzazione o del recupero presuppone la preventiva autorizzazione al loro svolgimento per cui la sua mancanza dovrebbe costituire presupposto per la cancellazione almeno per i dirigenti tenuto conto del fatto che -ex art. 28 dei contratti integrativi della dirigenza medica-veterinaria $\mathrm{e}$ di quella sanitaria, professionale, tecnica ed amministrativa sottoscritti il 10 febbraio 2004- essi sono apprezzabili ai citati fini esclusivamente se conseguenti a servizi di guardia e pronta disponibilità ovvero ad altre attività non programmabili riteniamo opportuno sottolineare che:

- il riconoscimento è da escludere quando essi sono utilizzati per:

- raggiungere gli obiettivi concordati con il singolo collaboratore,

- assicurare la copertura di turni di servizio predefiniti per lo svolgimento delle attività ordinarie, rilevando che se essi sono dovuti a carenze di organico le ore prestate vanno considerate come straordinario;

- il recupero, di norma disciplinato dai contratti integrativi aziendali, è soggetto comunque alla esplicita richiesta degli interessati e va consentito in tempi ravvicinati, a seguito di diretta e responsabile valutazione da parte del direttore circa l’inesistenza di necessità di servizio.

\subsection{Servizi di guardia e pronta disponibilità ${ }^{(1.2)}$}

La guardia medica -ex art. 16 del ccnl sottoscritto il 3 
novembre 2005- è mirata ad assicurare nelle ore notturne e nei giorni festivi la continuità assistenziale piuttosto che le emergenze/urgenze delle strutture complesse di nefrologia e/o dialisi. Essa:

- è svolta all'interno del normale orario di lavoro e, ove non possibile, tramite ricorso al lavoro straordinario se festiva, in orario o fuori orario di lavoro se notturna; in quest'ultimo caso da diritto alla corresponsione sia dello specifico compenso definito contrattualmente che dell'indennità notturna;

- coinvolge tutti i dirigenti medici della struttura, fatta eccezione per il suo direttore;

- è organizzata dall'azienda cui spetta darne comunicazione alle OO.SS. interessate per consentire loro di richiedere di procedere alla concertazione dei relativi criteri generali.

La pronta disponibilità -ex successivo art. 17 del ccnl richiamato- è finalizzata ad affrontare le situazioni di emergenza/urgenza non fronteggiabili con la guardia ovvero in carenza di presenza attiva medica. Essa:

- è caratterizzata dalla immediata reperibilità del medico, il quale è tenuto a raggiungere la struttura nel tempo definito in sede di contrattazione integrativa aziendale (di solito 30' dalla chiamata), essendosi posto nelle condizioni di ricevere comunque la chiamata;

- può essere sostitutiva o integrativa della guardia;

- deve essere limitata ai soli periodi notturni e festivi;

- deve avere, di norma, durata di dodici ore o comunque non inferiore a 4 ;

- deve, di norma, coinvolgere i medici per un massimo di 10 turni al mese, essendone consentito il coinvolgimento per due turni continuativi esclusivamente durante le giornate festive;

- coinvolge tutti i dirigenti medici della struttura, fatta eccezione per il suo direttore il cui coinvolgimento dovrebbe essere limitato ai soli turni di reperibilità integrativa;

- comporta la corresponsione dell'indennità nell'entità definita contrattualmente per ogni turno intero che va ridotta proporzionalmente e maggiorata del $10 \%$ in caso di durata inferiore alle 12 ore;

- comporta il riconoscimento delle ore prestate in seguito a chiamata come lavoro straordinario e quindi la loro remunerazione piuttosto che il loro recupero nel caso in cui le esigenze di servizio lo consentano;

- comporta, nel caso sia svolta in giornata festiva, il diritto a un giorno di riposo compensativo senza riduzione comunque dell'orario settimanale dovuto.

\subsection{Partecipazione ai procedimenti e provvedimenti di- sciplinari $^{(1,3)}$}

Premesso che l'istituto va attuato nel rispetto dei codici disciplinari aziendali, rileviamo che ciascun direttore -ex art. 55-bis del D.L.gs. n. 165/2001, come novellato dal D.Lgs. n.150/2009:

a. è tenuto tra l'altro a:

a.1. contestare - senza indugio e comunque non oltre 20 giorni dalla notizia di comportamenti punibili con la sanzione massima della sospensione dal servizio con privazione della retribuzione fino a un massimo di 10 giorni - l'addebito all'interessato convocandolo contestualmente per il contradditorio a difesa almeno 10 giorni prima della data individuata per lo svolgimento, comunicandogli la possibilità di giovarsi dell'assistenza di un procuratore ovvero di un rappresentante del sindacato;

a.2. svolgere il procedimento e concluderlo con l'archiviazione o l'irrogazione della sanzione entro 60 giorni dalla contestazione dell'addebito, salvo proroga in misura corrispondente nel caso di differimento superiore a 10 giorni del termine di difesa per impedimento del collaboratore, dovendo -ex successivo art. 55-ter- proseguirlo e concluderlo, a modifica di quanto avveniva in passato, anche quando per gli stessi fatti sia pendente procedimento penale;

a.3. trasmettere gli atti relativi al procedimento che comporti una sanzione superiore a quella indicata sotto la lettera "a.1" all'ufficio aziendale competente per i procedimenti disciplinari entro 5 giorni dalla loro conoscenza, dandone comunicazione allinteressato;

a.4. effettuare ogni comunicazione tramite posta elettronica certificata (se in possesso dell'interessato), consegna a mano, raccomandata con ricevuta di ritorno;

b. deve essere consapevole che:

b.1. i termini di cui alle precedenti lettere "a.1" ed "a.3" non sono procrastinabili;

b.2. l'omissione o il ritardo della contestazione, senza giustificato motivo, comportanti il mancato esercizio o la decadenza dell'azione disciplinare e valutazioni irragionevoli o manifestamente infondate sull'insussistenza dell'illecito disciplinare gli comportano ex comma 3 dei successivo art. 55-sexies- la sospensione dal servizio con privazione della retribuzione per un periodo temporale proporzionato alla gravità dell'infrazione non perseguita comunque non superiore a 3 mesi in relazione ad infrazioni sanzionabili con il licenziamento e, in aggiunta, la mancata attribuzione della retribuzione di risultato per un importo pari a quello spettante per il doppio della 
durata della sospensione previo svolgimento, a nostro avviso, di specifico procedimento disciplinare;

c. può acquisire da altre amministrazioni pubbliche informazioni e/o documenti rilevanti per la definizione del procedimento, invariati i termini per avviarlo e concluderlo;

d. deve avere presente che l'interessato, se non intende presentarsi per il contradditorio, può formalizzare, in caso di grave ed obiettivo impedimento, motivata istanza - essendogli stata comunicata la sua irripetibilità - per esercitare la difesa, prendere visione degli atti istruttori, indicare il numero di fax suo o del suo procuratore dove inviare le comunicazioni successive.

A conclusione dell'approfondimento si rileva che:

- l'art. ¿zý đeí ccní đeí personaíe đeí comparto, reíativo al quadriennio 1994/1997, come modificato dall'art. 12 del ccnl relativo al quadriennio 2002/2005, ha indicato le sanzioni in cui detto personale può incorrere e l'art. 13 di quest'ultimo contratto, come modificato dall'art.

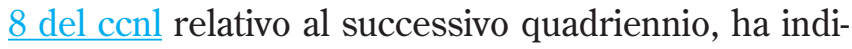
cato i criteri generali da osservare per la determinazione della sanzione da applicare ai comportamenti in esso esplicitati, fermo restando il rispetto dei principi di gradualità e proporzionalità;

- gli articoli 7 ed 8 dei ccccnnll per la sequenza contrattuale della dirigenza medica-veterinaria e di quella sanitaria, professionale, tecnica ed amministrativa sottoscritti il 17 ottobre 2008 hanno indicato rispettivamente le sanzioni in cui possono incorrere i dirigenti ed i criteri generali da osservare per la determinazione delle sanzioni da applicare ai comportamenti in essi esplicitati, fermo il rispetto dei principi di gradualità e proporzionalità.

\section{7. "Partecipazione alle procedure per l'affidamento de-} gli incarichi ai collaboratori medici” ${ }^{(1,4)(2,2)}$

Premesso che:

- ex combinaio ảisposio ảegii ar iicoli 15 , comma 4, e 15 . ter, comma 1, del D.Lgs n. 502/1992 e smi ai collabo. ratori sono attribuibili incarichi professionali di base ovvero, dopo cinque anni di attività con valutazione positiva, incarichi professionali di alta specializzazione, consulenza, studio e ricerca, verifica e controllo, ispettivi piuttosto che di direzione di struttura semplice nel rispetto delle modalità definite con i contratti collettivi nazionali;

- l'art. 51 del ccnl relativo al quadriennio 1994-1997 come modificato dall'art. 26 del ccnl relativo al successivo quadriennio ha indicato criteri e parametri da osservare per la definizione da parte della singola azienda della graduazione delle posizioni;

- gli articoli 27 e 28 del ccnl relativo al quadriennio 19982001 hanno individuato rispettivamente le "Tipologie di incarico" conferibili ai dirigenti medici e le modalità di "Affidamento e revoca degli incarichi dirigenziali. Criteri e procedure", procediamo all'esplicitazione dei comportamenti che ogni direttore è opportuno ponga in essere al fine di partecipare attivamente ed oggettivamente - cioè con esclusivo riferimento ad elementi assolutamente meritocratici quindi non influenzati da condizionamenti ispirati, ad esempio, a clientelismo, parentela, appartenenza politica e/o sindacale - alle procedure per l'affidamento degli incarichi ai collaboratori medici, rilevando che:

- diversamente non potrà evitare l'insorgere né di conflittualità all'interno della struttura, né di resistenza e scarsa/mancata collaborazione per il raggiungimento degli obiettivi della stessa;

- il D.Lgs. n. 150/2009 precisa:

- all'art. 40, lettera a), che "ai fini del conferimento di ciascun incarico di funzione dirigenziale si tiene conto, in relazione alla natura e alle caratteristiche degli obiettivi prefissati ed alla complessità della struttura interessata, delle attitudini e delle capacità professionali del singolo dirigente, dei risultati conseguiti in precedenza nell'amministrazione di appartenenza e della relativa valutazione";

- all'art. 20 le progressioni di carriera sono strumenti idonei a premiare il merito e la professionalità;

- il citato art. 28 del ccnl ha:

a. disposto che sia gli incarichi professionali di base che gli incarichi professionali e di struttura semplice, dopo 5 anni di attività con valutazione positiva, siano conferiti, su proposta del direttore, dall'azienda con atto scritto e motivato che ad evitarne l'impugnazione dovrà, a nostro parere, tenere conto delle motivazioni su cui si fonda la proposta;

b. previsto che l'attribuzione debba avvenire, nel rispetto dei criteri e delle procedure preventivamente definiti in sede aziendale previa concertazione con le OO.SS. interessate, tenendo conto:

b.1. dell'esito delle valutazioni annuali e triennali, cui sono soggetti tutti i dirigenti, concernenti le attività professionali svolte $\mathrm{e}$ i risultati raggiunti rispetto agli obiettivi assegnati;

b.2. della natura e caratteristiche del programma da realizzare;

b.3. delle attitudini personali e delle capacità professionali del singolo interessato relative sia alle conoscenze specialistiche che all'esperienza acquisita in precedenti incarichi. 


\subsection{Caratterizzazione della gestione}

Il direttore per realizzare una gestione dei collaboratori caratterizzata dagli elementi poc'anzi esplicitati oltre a dotare la struttura di un sistema tempestivo e trasparente di comunicazione interna deve:

- possedere autorevolezza/leadership e capacità decisionali;

- essere di esempio;

- essere in grado di:

- valutare ed esplicitare l'importanza del contributo di ciascuno al raggiungimento degli obiettivi della struttura;

- apprezzare quanti in ogni situazione e nei confronti di chicchessia dimostrano piena lealtà assicurando che li supporterà/difenderà se commettono errori non comportanti loro responsabilità dirette;

- perseguire e sollecitare la realizzazione di comportamenti rispettosi della dignità dei pazienti;

- dimostrare di agire con correttezza, equità e trasparenza nelle sue numerose incombenze e, in particolare, nelle valutazioni da cui derivano benefici economici o di carriera e nel gestire il delicato sistema dei procedimenti e provvedimenti disciplinari;

- essere consapevole che le riferite caratterizzazione costituiscono base irrinunciabile anche per:

- disporre di collaboratori in possesso di professionalità attualizzata rispetto al ruolo rivestito ed alle funzioni da svolgere in quanto consapevoli del fatto che dal loro sviluppo dipende il raggiungimento degli obiettivi di efficienza, economicità, qualità, appropriatezza ed efficacia propri della struttura e, quindi, di riflesso, dell'azienda di appartenenza;

- ottenere la piena responsabilizzazione dei collaboratori rispetto al raggiungimento dei risultati prefigurati per il singolo e per la struttura,

rilevando che a lui spetta stabilire caso per caso se realizzare prima i comportamenti utili a favorire lo sviluppo e poi la responsabilizzazione o viceversa.

Ciò premesso di seguito ci accingiamo ad esplicitare $\mathrm{i}$ comportamenti che riteniamo debba realizzare ogni $d i$ rettore per raggiungere i riferiti irrinunciabili obiettivi consapevole della necessità del pieno coinvolgimento dei collaboratori e della particolare onerosità del loro sviluppo.

\section{Lo sviluppo dei collaboratori}

\section{Aspetti generali ${ }^{(1.5)}$}

Lo sviluppo degli operatori, fattore irrinunciabile per assicurare il più alto livello qualitativo delle prestazioni da erogare, ha assunto particolare importanza fin dal momento in cui il Legislatore dopo aver precisato all'art. 2 della legge 23 dicembre 1978, n. 833, "Istituzione del servizio sanitario nazionale" che il conseguimento delle finalità del servizio stesso avrebbe dovuto essere assicurato, tra l'altro, mediante la formazione professionale e permanente nonché l'aggiornamento scientifico-culturale del personale ha disciplinato con gli articoli 16-bis, 16-quater e 16-quinquies del D.Lgs. n. 502/1992 come novellato dal D.Lgs. n. 229/1999, rispettivamente:

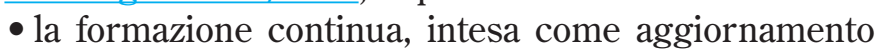
professionale e formazione permanente,

- l'incentivazione alla partecipazione alle attività della formazione,

- la formazione manageriale.

Ciononostante segnaliamo che le disposizioni legislative richiamate non devono far dimenticare l'importante ruolo che al riguardo devono sempre più assumere, come vediamo di seguito:

- la cosiddetta "Clinical Competence",

- l'importante ruolo che al riguardo devono assumere

- il cosiddetto "audit professionale" e

- la "omogeneizzazione comportamenti clinici”.

\section{La Clinical Competence}

Nella letteratura la Clinical Competence concerne i processi di certificazione professionali internazionali e rappresentata non un risultato raggiunto ma una abitudine ad apprendere per tutto il corso della vita professionale ${ }^{(3)}$ cioè ad attualizzare le conoscenze onde renderle adeguate al ruolo e alle funzioni cui si è chiamati durante i vari periodi del rapporto di lavoro. Tale elemento va tenuto presente da ciascun collaboratore, soprattutto medico, considerando che, come è scontato, l'attività specialistica nefrologica si caratterizza per una professionalità inserita in un contesto di rete di fornitura delle prestazioni, dove il singolo specialista difficilmente agisce in completa autonomia ma abitualmente collabora all'interno di un sistema integrato. In questo contesto la Clinical Competence nefrologica non ha solo un aspetto individuale collegato alla garanzia di qualità delle cure o delle prestazioni fornite dal singolo professionista ma interagisce con quella della intera équipe e si integra in essa, configurandosi quindi come Clinical Competence ${ }^{(4)} \mathrm{di}$ équipe correlata alla "mission" delle diverse tipologie di Centro previste dalla rete nefrologica del territorio per cui si costituisce, come Qualità Professionale, parte integrante del Sistema Qualità di ogni Unità Operativa afferente alle rete ${ }^{(5)}$.

Essa secondo la SINCERT(6):

- definisce i requisiti minimi previsti e intesi come percor- 
so formativo finalizzato ad acquisire la capacità di fare in sicurezza una specifica attività assistenziale;

- può essere definita dalle istituzioni diventando requisito obbligatorio (alcune Regioni hanno definito $i$ requisiti in relazione all'accreditamento) e/o da società scientifiche;

- presuppone la definizione (utilizzando riferimenti istituzionali, di società scientifiche e/o della propria esperien$z a)$ dei requisiti da parte del singolo direttore nella sua funzione di responsabile organizzativo;

- costituisce presupposto indispensabile per definire i bisogni di formazione e un piano di addestramento specifico. A ciò aggiungasi che essa da un lato si fonda, se riferita all'équipe, sulla sommatoria delle competenze dei singoli professionisti per cui rende possibile l'assegnazione ad ognuno dei "privilegi”, intendendo con questo termine gli atti medici "skills" che esso è autorizzato a svolgere all'interno della organizzazione dove opera e da un altro lato ${ }^{(7,8)}$ comporta valutazioni affidate esclusivamente ad indicatori e strumenti in grado di ridurre la variabilità di giudizio individuale riferite peraltro solo alle performance ottenute.

A ciò aggiungiamo che sulla base anche delle disposizioni dettate dai richiamati decreti legislativi ogni direttore dovrebbe essere indotto a:

- stimolare nel singolo collaboratore il desiderio ad acquisire le informazioni e le conoscenze utili sia a realizzare le rispettive aspirazioni di carriera -quindi un migliore stato economico e sociale- che a mettere a disposizione della struttura le rispettive potenzialità onde questa possa fornire, a chi la frequenta per soddisfare i propri reali bisogni di salute, prestazioni e servizi di alto livello qualitativo, appropriati, efficaci, rispettosi anche della loro dignità in un irrinunciabile contesto di efficiente ed economico utilizzo delle risorse disponibili;

- formalizzare proposte, in sede di definizione del budget di struttura, idonee a far acquisire ai collaboratori le conoscenze tecnico/professionali e, a volte, anche gestionali necessarie per l'attualizzazione di quelle già possedute onde rendere più facilmente raggiungibili gli obiettivi prefigurati nel budget avendo presenti:

- le reali potenzialità del singolo;

- la necessità che gli interventi formativi proposti siano recuperati all'interno del programma delle attività formative anche nel caso in cui gli stessi o loro parte potranno essere realizzati in loco.

\section{L'audit professionale}

L'audit professionale, ovverosia clinico, è attualmente l'unico sistema di valutazione della qualità professionale ${ }^{(9)}$ ed è un approccio alla verifica che si focalizza su specifici problemi clinici e assistenziali o aspetti della pratica corrente. Esso che è definito dal National Health Service (NHS) inglese come il "processo di controllo della qualità che ha l'obiettivo di migliorare l'assistenza al paziente attraverso una revisione sistematica della pratica clinica rispetto a criteri precisi" nella versione finale sottolinea l'importanza del processo di miglioramento: "further monitoring is used to confirm improvement in healthcare delivery" ${ }^{(10)}$.

Esso è caratterizzato dalla 'professionalità' dell'iniziativa, che si esprime con alcuni ingredienti tipici - competenza clinico-assistenziale specifica dei partecipanti, confidenzialità dei risultati, oggetto fortemente connesso alla qualità dei professionisti e si basa tra l'altro, fermo restando la trattazione sistematica fattane da Esposito ${ }^{(11)}$ sulle seguenti premesse fondamentali:

a) l'indicatore e lo standard devono essere validati dalla letteratura;

b) l'acquisizione del dato deve essere facile da ottenere e possibilmente estraibile da un sistema informatico;

c) deve coinvolgere tutto il personale;

d) presuppone il cambiamento della pratica clinica se ritenuta essenziale per il miglioramento dei risultati. $\mathrm{Al}$ riguardo segnaliamo che la SIN incoraggia l'uso dell'audit per la valutazione e revisione della pratica clinica ${ }^{(12)}$ : "PM3: Il programma si serve delle informazioni ricavate dalle misure per supportare, migliorare e/o validare il processo decisionale clinico. Non è opportuno pertanto scegliere indicatori che misurano processi e/o esiti già ottimali. Ogni 6 mesi si effettua un audit sulle misure implementate e si analizzano $i$ dati fuori misura individuando i pazienti ed apportando le modifiche necessarie a migliorarne l'output".

\section{L'omogeneizzazione comportamenti clinici}

I comportamenti clinici si basano sulla appropriatezza e in particolare sulla medicina basata sulle prove di efficacia. In nefrologia purtroppo non sono molti i comportamenti clinici basati su prove cliniche forti. Un'indagine di Strippoli ${ }^{(13)}$ ed altre pubblicazioni ${ }^{(14)}$ mostrano che molti comportamenti non sono basati su evidenze forti e che invece l'area di incertezza è molto ampia. Spetta al singolo diretttore tracciare le linee di quella che si può definire strategia di trattamento che, omogeneizzando i comportamenti, comporta una più efficace continuità della cura. A titolo di esempio possiamo ricordare il diverso comportamento nei confronti del trattamento dietetico, dell'uso della bioimpedenza, della raccolta urinaria con determinazione della fosfaturia e sodiuria, dell'uso assiduo o saltuario degli indici di efficienza dia- 
litica e molti altri, senza considerare le varie strategie terapeutiche in varie occasioni come ad esempio nelle glomerulonefriti e nel trapianto.

Le basi di tale atteggiamento determinano poi la scelta dell'indicatore che potrebbe essere usato nell'audit. Del tutto obsoleto ed arcaico risulta il ricorso alla visita collegiale al letto del paziente. Le nuove norme sull'autonomia professionale dei dirigenti medici e la scomparsa del mansionario infermieristico hanno decretato la fine di una usanza spesso prevaricante, ma sostanzialmente autoreferenziata e basata sulla autorità ${ }^{(15)}$. Ben diverso invece il ricorso ad incontri regolari in sala medici con le cartelle dei pazienti in cui si esaminano le strategie, le risorse, le opzioni terapeutiche e i risultati. Il "discussant" può essere il dirigente medico che ha in carico il paziente o lo specializzando che così impara a porsi di fronte a problemi reali e ad esporli in maniera corretta, semplice ma sintetica. $\grave{E}$ anche utile per tutta l'équipe che si cimenta in discussioni aperte, spesso non possibili al letto del malato, davanti ai vari computer dove sono basati sia i dati biochimici ma ancor più spesso le immagini delle indagini diagnostiche. In questa sede il direttore potrà far valere la sua scienza e/o esperienza ma soprattutto dovrà gestire la discussione, i conflitti, far rispettare i tempi e soprattutto arrivare ad una conclusione condivisa.

\section{La responsabilizzazione dei collaboratori( ${ }^{(1.6)}$}

\section{Aspetti generali}

Premesso che, come accennato in precedenza, la disponibilità dei collaboratori a partecipare attivamente al raggiungimento degli obiettivi predefiniti nel budget di struttura presuppone la loro piena responsabilizzazione e che il raggiungimento dell'obiettivo presuppone che essi attualizzino la rispettiva professionalità onde poter adempiere correttamente le competenze correlate al ruolo ed alle funzioni di cui ciascuno è titolare, ivi compresa la disponibilità a contribuire alla realizzazione della funzionalità della struttura, di seguito individuiamo i comportamenti finalizzati a perseguirne la responsabilizzazione sottolineando che essa:

- presuppone il loro consapevole coinvolgimento e la realizzazione di interventi idonei a perseguirlo, avendo presente che alcuni sono di competenza del direttore mentre altri vanno realizzati, su sua sollecitazione, da soggetti terzi;

- va rivitalizzata continuamente, essendo raramente frutto di auto convincimento, tramite interventi ad hoc onde evitare l'insorgere di periodi di demotivazione;

- ha difficoltà a concretizzarsi nei casi in cui:

- il collaboratore nutra scarsa fiducia nei soggetti cui spetta realizzare gli specifici riferiti interventi; - gli interventi prefigurati non siano realizzati;

- il direttore operi in maniera ritenuta iniqua principalmente nella valutazione dei collaboratori, nell'attribuzione dei benefici, soprattutto economici, nella valutazione di comportamenti analoghi censurabili sotto l'aspetto disciplinare;

- il singolo collaboratore reputi che il mancato raggiungimento degli obiettivi personali dia comunque luogo a sanzioni di scarsa rilevanza anche economica, in presenza soprattutto di somme di esigua entità e/o di loro corresponsione a pioggia;

- si configura come presupposto indispensabile per la fidelizzazione del singolo collaboratore rispetto alla struttura e, quindi, all'azienda di appartenenza.

2. Elementi indispensabili alla realizzazione del coinvolgimento

Le riferite riflessioni e la consapevolezza che il raggiungimento della performance individuale e degli obiettivi della struttura sia possibile solo tramite:

- un utilizzo efficiente ed economico delle risorse;

- il massimo livello di qualificazione delle sue attività e la loro appropriatezza ed efficacia;

- l'adempimento corretto da parte di ogni collaboratore delle proprie competenze professionali e, ove attribuite, gestionali;

ci inducono a pensare che acquisiscano particolare importanza per indurre il singolo collaboratore ad operare quotidianamente per il raggiungimento dei riferiti obiettivi:

- la disponibilità di un ambiente lavorativo oltre che dotato di procedure operative idonee e di tecnologie adeguate, anche permeato di clima collaborativo e capace di generare uno spirito di emulazione tra chi vi opera;

- l'utilizzazione adeguata rispetto alla professionalità posseduta;

- il soddisfacimento delle rispettive aspettative comparativamente con la professionalità e le aspettative degli altri operatori assegnati alla struttura;

- la consapevolezza di poter operare in piena autonomia professionale, fermo il rispetto degli indirizzi del $d i$ rettore e la coerenza con gli obiettivi complessivi della struttura, avendo certezza di ricevere adeguati supporti nel caso incontri difficoltà nello svolgimento dei compiti assegnatigli,

- il riconoscimento da parte soprattutto del direttore e, magari, da parte di tutti gli altri operatori della struttura del ruolo svolto al suo interno e dell'apporto reale fornito al raggiungimento dei suoi obiettivi;

- la certezza dell'attribuzione dei benefici, economici e non, esclusivamente previe valutazioni meramente 
meritocratiche e della uniforme valutazione di comportamenti analoghi rispetto alla loro censurabilità sotto l'aspetto disciplinare;

- la disponibilità tempestiva di informazioni, ad esempio, sul cambiamento degli obiettivi della struttura, sulla sua organizzazione e/o sulle modalità di suo funzionamento, sui colleghi chiamati a nuove competenze e/o responsabilità.

Pur convinti della non esaustività dell'elencazione, provvediamo a terminarla ritenendo da un lato che ogni direttore potrà arricchirla sulla base sia delle sue esperienze e conoscenze che delle immancabili peculiarità di ciascuna struttura complessa di nefrologia e/o dialisi e da un altro lato di aver fornito un ampio panorama degli elementi idonei a favorire il pieno, consapevole e convinto coinvolgimento di ciascun collaboratore per quanto concerne la sua piena responsabilizzazione rispetto al raggiungimento degli obiettivi predefiniti per sé e per la struttura complessivamente considerata.

3. La responsabilizzazione quale presupposto per la fidelizzazione alla struttura ed all'azienda di appartenenza

L'affermazione, già esplicitata, secondo cui la responsabilizzazione costituisce il presupposto per la fidelizzazione del singolo operatore alla struttura ed all'azienda di appartenenza dovrebbe inevitabilmente comportare, sulle base delle considerazioni fin qui sviluppate, che ciascun collaboratore - sentendosi in dovere di contribuire attivamente alla operatività complessiva della struttura e orgoglioso dell'appartenenza alla stessa e del ruolo ricoperto al suo interno - percepisca di essere un tutt'uno con essa e quindi con l'azienda - configurandosi la struttura come una delle tante articolazioni organizzative di cui l'azienda si avvale per raggiungere la mission affidatele - ed indurlo, data la situazione di simbiosi accennata, a realizzare comportamenti di grande importanza per assicurare piena funzionalità di entrambe consistenti principalmente nella disponibilità a:
- attuare le direttive/disposizioni del direttore e quindi agire in coerenza con gli indirizzi da lui formalizzati per assicurare la piena funzionalità della struttura;

- attualizzare periodicamente la propria professionalità;

- formalizzare suggerimenti e proposte finalizzati, esempio, a migliorare l'ambiente lavorativo piuttosto che a razionalizzare i percorsi utilizzati;

- evitare resistenze rispetto ai cambiamenti disposti dal direttore, ferma restando la doverosità di costui di procedere, dopo valutazione con i collaboratori delle conseguenze derivanti da essi, a definirne i contenuti previa esplicitazione delle motivazioni su cui si fondano;

- essere rispettosi nei confronti dei superiori e dei colleghi;

- rispettare la dignità di quanti si rivolgono alla struttura per soddisfare i rispettivi reali bisogni di salute, indipendentemente dallo stato sociale, dalla religione, dalla nazionalità di questi ed essendo conscio che $\mathrm{i}$ comportamenti conseguenti devono essere comunque permeati di umanità;

- agire con cortesia nei confronti dei parenti dei pazienti e di coloro che chiedono informazioni, esempio, sull'organizzazione delle attività della struttura piuttosto che sulle modalità da osservare per ricevere le prestazioni.

\author{
Indirizzo degli Autori: \\ Dr. Gianfranco Carnevali \\ Via XX Settembre 124 \\ 06124 Perugia \\ g.carnevali@live.it \\ www.gianfrancocarnevali.com \\ Dr. Giuseppe Quintaliani \\ Via Maturanzio 31 \\ 06124 Preugia \\ quintaliani@yahoo.it
}

\section{Bibliografia}

1. Carnevali G. Il direttore di struttura complessa sanitaria (e non). Competenze, responsabilità, gestione delle risorse umane. Roma: Edizioni “Panorama della Sanità”, 2011.

(1.1) pagg. 73-77; 141-144.

(1.2) pagg. 122-127.

(1.3) pagg. 85-113.

(1.4) pagg. 77-82.
(1.5) pagg. 129-133; 145-155.

(1.6) pagg. 157-162.

2. Carnevali G, Greco M. Il rapporto di lavoro dei Medici-Chirurghi e degli Odontoiatri con le aziende del Servizio Sanitario Nazionale. Torino: G.C. Edizioni Medico Scientifiche, 2006.

(2.1) pagg. 33-34.

(2.2) pagg. 53-56.

3. Leach DC. Competence is a habit. JAMA 2002; 287: 243-4. 
4. Palsson R, Kellet J, Lindgren S, Merino J, Semple C, Saereni D. Core competencies of the European internist: A discussion paper. Eur J Intern Med 2007; 18.

5. Bonaldi A. Come migliorare la qualità professionale. G Ital Nefrol 2002; 19(S-21): S14-520.

6. SINCERT. Prescrizioni per l'accreditamento degli Organismi operanti la valutazione e certificazione dei sistemi di gestione per la qualità nel settore EA 38 "Sanità e altri servizi sociali”. SINCERT RT-04, Rev. O3/2004.

7. Rosner MH, Bern JS, Parker M, Tolwani A, Bailey J, Di Giovanni S, Lederer E, Norby S, Plumb TJ, Qian Q, Yeun J, Hawley JL, Owens S. (The ASN In-Training Examination Committee). Development, Implementation, and Results of the ASN In-Training Examination for Fellows. CJASN 2010; 2: 328-34.

8. Rethans JJ, Norcini JJ, Baron Maldonaldo M, et al. The relationship between competence and performance: implications for assessin practice performance. Med Educ 2002; 36 : 901-09.

9. More clinical audits are key to improving quality of care, says NHS audit chief. BMJ 2009; 339 doi: 10.1136(bmj. b3878(Published 21 September 2009).

10. National Institute for Clinical Excellence, CHI, Royal College of Nursing, University of Leicester. Principles fox Best Practice in Clinical Audit. In Oxon, UK: Radcliffe Medican Press Ltd, 2002.
11. Esposito P, Dal Canton A. The clinical audit: a strategy to improve quality of care. G Ital Nefrol 2011; 28(2): 219-25.

12. Quintaliani G, et al. Standard PM3 Chronic kidney disease certification process manual by the Italian Society of Nephrology. J Nrphrol 2009; 22(5): 565-70.

13. Di Micco L, Manno C, Cianciaruso B, Stippoli G. The usefulness of Cochrane systematic reviews in nephrology. G Ital Nefrol 2009; 26(1): 38-54.

14. Donald A. Molony. Evidence Based Nephrology. M.D. e Jonathan C. Craig Editor.

15. http://www.youtube.com/watch?v=9e13jc7tGVA.

\section{Letture consigliate}

- Ferrè L.-Longo N. (a cura di). Ruoli e competenze nella sanità. Roma: Edizioni "Panorama della Sanità", 2011.

- Franci E, Dominijanni M, Mercone A. La gestione delle risorse umane in sanità. In "Mondo sanitario", 2007; n. $5 / 6$.

- Gandini T. Competenze. La valorizzazione del patrimonio umano in sanità. Milano: Franco Angeli, 2005.

- Sebastiani V, Quercioli C. La risorsa umana: valore che genera valore. In "Mondo sanitario”, 2007; n. 7/8.

La versione on-line del presente articolo disponibile sul sito della Società Italiana di Nefrologia www.sin-italy.org contiene collegamenti attivi alla documentazione legislativa citata nel testo 